\title{
Time-Scale Invariant Audio Data Embedding
}

\author{
Mohamed F. Mansour \\ Department of Electrical and Computer Engineering, University of Minnesota, Minneapolis, MN 55414, USA \\ Email: mmansour@ece.umn.edu
}

\begin{abstract}
Ahmed H. Tewfik
Department of Electrical and Computer Engineering, University of Minnesota, Minneapolis, MN 55414, USA Email: tewfik@ece.umn.edu
\end{abstract}

Received 31 May 2002 and in revised form 22 December 2002

\begin{abstract}
We propose a novel algorithm for high-quality data embedding in audio. The algorithm is based on changing the relative length of the middle segment between two successive maximum and minimum peaks to embed data. Spline interpolation is used to change the lengths. To ensure smooth monotonic behavior between peaks, a hybrid orthogonal and nonorthogonal wavelet decomposition is used prior to data embedding. The possible data embedding rates are between 20 and 30 bps. However, for practical purposes, we use repetition codes, and the effective embedding data rate is around $5 \mathrm{bps}$. The algorithm is invariant after time-scale modification, time shift, and time cropping. It gives high-quality output and is robust to mp3 compression.
\end{abstract}

Keywords and phrases: data embedding, broadcast monitoring, time-scale invariant, spline interpolation.

\section{INTRODUCTION}

In this paper, we introduce a new algorithm for high-capacity data embedding in audio that is suited for marketing, broadcast, and playback monitoring applications. The purpose of broadcast and playback monitoring is primarily to analyze the broadcasted content and collect statistical data to improve the content quality. For this class of applications, the security is not an important issue. However, the embedded data should survive basic operations that the host audio signal may undergo.

The most important requirements of a data embedding system are transparency and robustness. Transparency means that there is no perceptual difference between the original and the modified host media. Data embedding techniques usually exploit irrelevancies in digital representation to assure transparency. For audio data embedding, the masking phenomenon is usually exploited to assure that the distortion due to data embedding is imperceptible. Robustness refers to the property that the embedded data should remain in the host media regardless of the signal processing operations that the signal may undergo.

The research work in audio watermarking can be classified into two broad classes: spread-spectrum watermarking and projection-based watermarking. In spread-spectrum watermarking, the data is embedded by adding a pseudo random sequence (the watermark) to the audio signal or some features derived from it. An example of spread-spectrum wa- termarking in the time domain was presented in [1]. The features used for data embedding include the phase of the Fourier coefficients [2], the middle frequency coefficients [3], and the cepstrum coefficients [4]. More complicated structures for spread spectrum watermarking (e.g., [5]) were proposed to synchronize the watermarked signal with the watermark prior to decoding. On the other hand, projectionbased watermarking is based on quantizing the host signal to two or more codebooks that represent the different symbols to be embedded. The decoding is done by quantizing the watermarked signal and deciding the symbol that corresponds to the codebook with minimum quantization error. Examples of this technique are described in $[6,7]$.

Signal synchronization is an important issue in watermark decoding. Loss of synchronization will result in random decoding even if the individual watermark components are extracted correctly. In this paper, we propose a new embedding algorithm that is automatically robust to most synchronization attacks that the signal may undergo.

The proposed algorithm is designed to be transparent and robust to most common signal processing operations. It is automatically invariant under time-scale modification (TSM), which is the most severe attack to most data embedding algorithm. In addition, it is robust to basic signal processing operations, for example, lowpass filtering, mp3 compression, and bandpass filtering. Also, the embedding algorithm is localized in nature, hence it is robust to synchronization attacks, for example, cropping and time shift. The idea of 


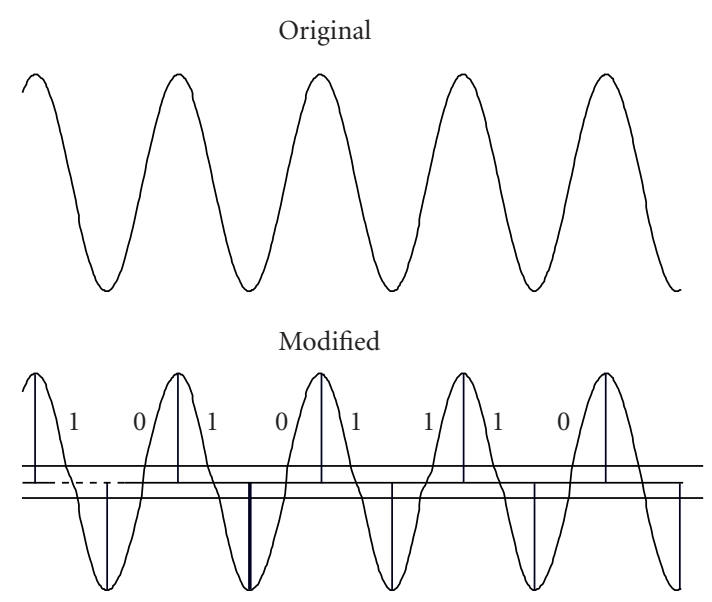

Figure 1: Embedding example.

the algorithm is to change the length of the middle segment between two successive peaks relative to the total length between the two peaks so as to be greater or less than a certain threshold to embed one or zero, respectively. Hence, if the signal is subject to TSM, then both the middle interval and the whole segment will change by the same factor leaving the ratio unchanged. Hence, the algorithm is automatically robust to TSM without need to rescale the signal. This work was first introduced in [8].

The average embedding capacity of the algorithm is 20 30 bps. However, due to practical issues that will be discussed in Section 3, the embedded data is encoded first with low code rate. The effective embedding rate drops to 4-6 bps.

The paper is organized as follows. Section 2 describes the basic idea of the embedding and extraction algorithms. Section 3 discusses several practical issues and the implementation details of the general ideas described in Section 2. In Section 5, the experimental results of the algorithm are given.

\section{ALGORITHM}

\subsection{Basic idea}

The intervals between a successive maximum and minimum pair are partitioned to $N$ segments of equal amplitude where $N$ is odd (typically $N=3$ or 5 ). If we have an exact linear behavior between the two extrema, then all the segments will be of equal size (up to a quantization error). For sinusoidallike segments, the outer segments tend to be longer than the inner ones because of the smaller slope at these segments.

If we assume that the total length of the intervals between the two peaks is $L$ and the length of each segment is $l_{i}$, then the basic idea of the algorithm is to control the ratio $l_{(N+1) / 2} / L$ to be greater or less than a certain threshold $\gamma$ to embed one or zero, respectively. The idea is illustrated in Figure 1.

Note that the smoothness of the signal is increased when it is lowpass filtered. This results in higher embedding capacity. However, we need to efficiently reconstruct the signal from the lowpass component. In our implementation, we

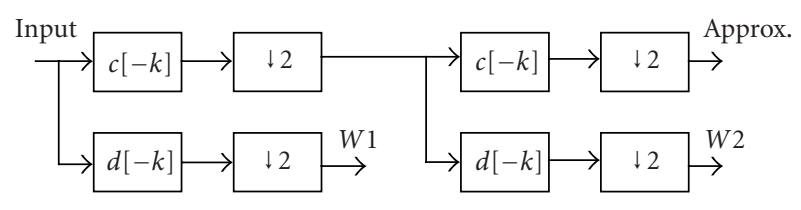

(a) Analysis stage.

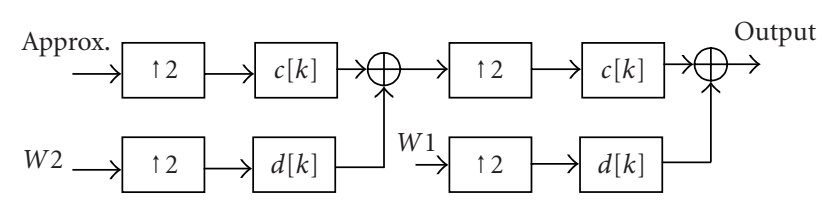

(b) Synthesis stage.

FIGURE 2: Orthogonal wavelet decomposition.

used a hybrid of orthogonal and nonorthogonal wavelet decompositions (as will be discussed in the next subsection) to satisfy the two requirements of smoothness and efficient reconstruction. The approximation signal at the coarsest scale is modified rather than the signal itself. The practicalities of choosing the possible intervals and selecting the threshold are discussed in Section 3.

\subsection{Hybrid orthogonal/nonorthogonal wavelet decomposition}

The required smooth behavior does not occur often in audio signals except for a set of single-instrument audio like a piano and a flute. For other composite audio signals, this requirement is hardly fulfilled. This greatly reduces the embedding rate if the original signal is used directly in embedding. Moreover, even if such a behavior exists, it is very vulnerable to distortion after compression. Hence, the direct audio signal is not a good candidate for data embedding.

In our implementation, we used a hybrid of orthogonal and nonorthogonal decompositions. These two types of decompositions are illustrated in Figures 2 and 3.

The orthogonal decomposition is an exact (nonredundant) representation of the signal. It involves subsampling after each decomposition stage. Hence, the approximation signal is not smoother than the original because of the frequency spread after subsampling. If a modification is done in the transform domain, then it is preserved after the inverse and the forward transform because it is nonredundant.

On the other hand, nonorthogonal wavelet decomposition does not involve subsampling after filtering at each scale, as illustrated in Figure 3. For our particular purpose, this decomposition has a two-fold advantage. First, the lengths are preserved so that the lengths at any scale are in one-to-one correspondence with the lengths at the finest scale. The second advantage is that the approximation signal at coarser scales is smoother than the signal at a finer scale.

However, nonorthogonal decomposition is redundant, that is, not every two dimensional signal is a valid transform. Hence modification in the transform domain are not guaranteed to be preserved if the inverse transform is applied. This 


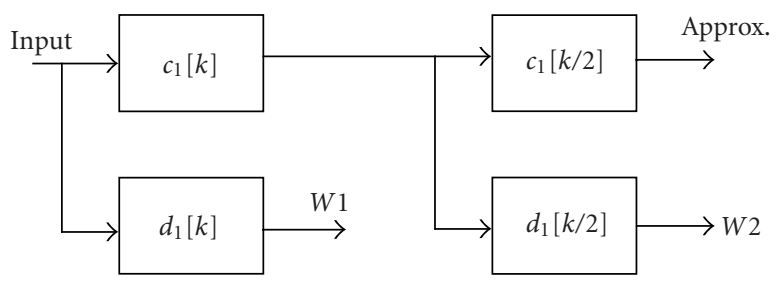

(a) Analysis stage.

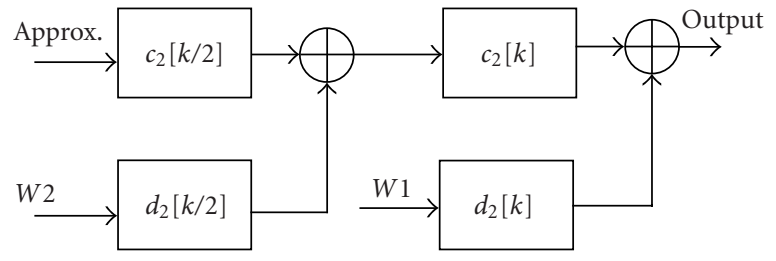

(b) Synthesis stage.

Figure 3: Nonorthogonal wavelet decomposition.

is more apparent if the modification is done at a sufficiently coarse scale. Hence, at most, two decomposition scales can be used to embed the data. However, this is not sufficient for robustness against lossy compression.

In Figure 4, we illustrate the ideas in the previous two paragraphs. The first $10^{3}$ samples of the original signals are plotted along with the approximation signal after three scales of nonorthogonal and orthogonal decompositions. We notice that the approximation signal after the nonorthogonal decomposition is much smoother.

In our system, the orthogonal decompositions is applied first. The resulting approximation signal is further decomposed using nonorthogonal decomposition. The orthogonal decomposition gives the required robustness against lossy compression but at the cost of reducing the interval lengths, that is, reducing the embedding rate. The nonorthogonal decomposition gives the required smooth behavior between peaks. Typically, one scale of orthogonal decompositions is used with two scales of nonorthogonal decompositions.

It should be mentioned that the filters of orthogonal and nonorthogonal decompositions need not be similar. Different bases can be used within the same framework. The optimality of choosing the wavelet basis for the problem is beyond the scope of this paper. However, experimental results show that different wavelet bases give very comparable results.

\subsection{The embedding algorithm}

The first step in the embedding algorithm is to apply a hybrid of orthogonal and nonorthogonal decompositions as discussed in the previous subsection. After decomposition, the approximation signal at the coarsest scale is used for embedding.

The next step is to change the relative length of the middle segment between two successive refined extrema to match the corresponding data bit. Let the total number of samples

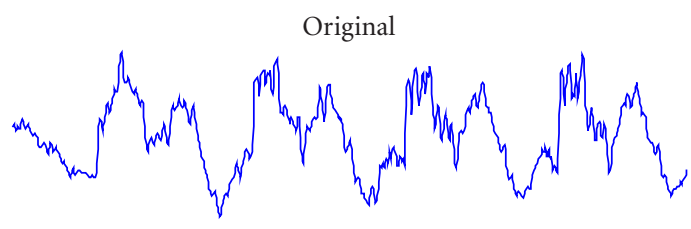

After nonorthogonal decomposition

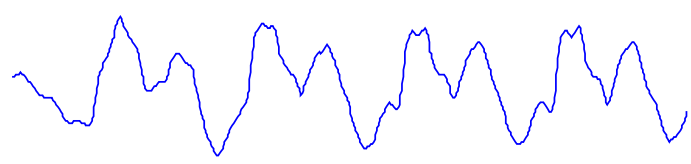

After orthogonal decomposition

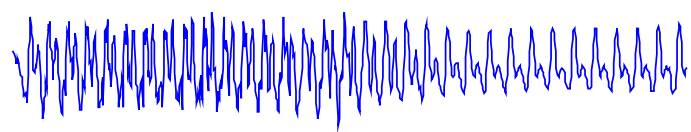

FIgUrE 4: Examples of orthogonal and nonorthogonal decompositions.

between the two extreme points be $L$ and let the length of the middle segment be $l$. Define $\alpha=l / L$ and the threshold $\gamma$. To embed one, $\alpha$ should be greater than $\gamma$, and vice versa. Spline interpolation is used to modify the lengths. An increase in the middle segments will reflect in a decrease in both of the outer segments, and vice versa, so as to keep the original length between the extrema unchanged. Some details for accelerating the convergence and improving the error performance are described in Section 3.3. The overall algorithm is illustrated in Figure 5.

The main difficulty with the algorithm described above stems from the redundancy of the nonorthogonal wavelet transform. Specifically, not all 2D functions are valid wavelet transform. Therefore, it is possible to end up with a nonvalid transform after modifying the coarsest scale of the signal. In particular, some previously used intervals may disappear or new intervals may arise. This causes a shift in the embedded sequence from one iteration to another and slows down the convergence. To partially fix this problem, a repetition code is used where each bit is repeated an odd number of times (typically five). The advantage of a repetition code is twofold. First, at the encoder side, it accelerates the convergence because a smaller number of intervals will need modification after peak deviation. Second, at the decoder side, it alleviates the problem of false alarms as will be discussed later. The transitions from zero to one and from one to zero are labeled as markers in the embedded sequence. These markers play a crucial role in synchronizing the data in the presence of false alarms and missed peaks as will be described in Section 4.2.

\subsection{The extraction algorithm}

The extraction algorithm is straightforward. The hybrid decomposition is applied as in the encoder. Then, the peaks 


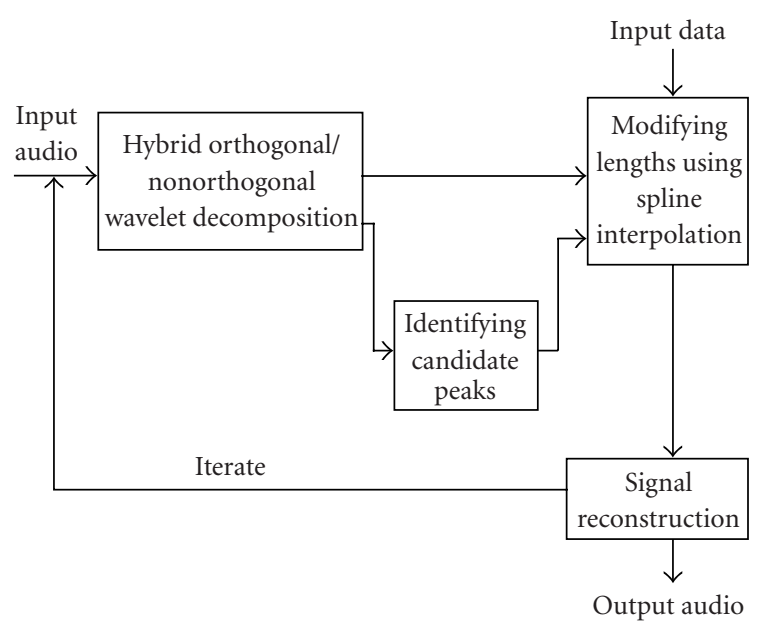

FIGURE 5: Embedding algorithm.

are picked and refined. For the refined intervals, the ratio $\alpha(=l / L)$ is calculated. If $\alpha>\gamma$, then decide one, otherwise decide zero. If a repetition code is used, then the majority rule is applied to decide the decoded bit.

This extraction algorithm works well with nice channels, which do not introduce false alarms or missed data, that is, channels with no synchronization problems. This type of channels is a good model for simple operations like volume change. However, if the audio signal undergoes compression or lowpass filtering, this ideal situation cannot be assumed and additional work has to be done to synchronize the data and remove the false alarms. The details of the practical decoding algorithm is discussed in Section 4.3.

\section{PRACTICAL ISSUES}

\subsection{Refining the extrema}

The careful selection of the extrema is an important issue in the algorithm performance. The objective here is to identify the pairs of successive extrema between which reliable embedding is possible. The first requirement is to choose the pairs with distance greater than a certain threshold. This threshold should guarantee that the middle segment and each of the outer segments contain at least two samples after modifying their lengths. The second requirement is that a refined peak should be a strong one in the sense that it should be significantly larger (or smaller) than its immediate neighbors. This is important to ensure that the peak will survive modifications and compression.

Here it is important to mention that adjacent peaks that are very close to each others and very close to their immediate neighbors are labeled as weak peaks. In our algorithm, weak peaks are not considered peaks at all and they are ignored if they exist between two successive strong peaks. Those weak peaks usually arise if the signal undergoes compression. Hence if they are treated as peaks candidates, they may lead to missed peaks.

\subsection{Threshold selection}

The selection of the threshold $\gamma$ is important for the quality of the output audio and the fast convergence at the encoder. To minimize the modifications due to changing the length of the middle interval, we first calculate the histogram of the ratio of the middle segment length to the interval length. Then we set the threshold as the median of the histogram. This is done offline only once using a large set of audio pieces.

\subsection{Modifying the lengths}

The embedding algorithm as described earlier requires modification of the lengths of interval segments. For example, assume we have a data bit of 1 , then $l / L$ should be greater than $\gamma$. Otherwise, the length $l$ of the middle segment should be increased to satisfy this inequality. The increment in the middle segment reflects in a decrement in both of the outer segments so as to preserve the original interval length. The process is reversed for embedding zero. The modification of all segments is performed via spline interpolation.

To improve the error performance and to give additional robustness against TSM, a guard band is built around the threshold so that all modified segments are at least two samples above or below the threshold value.

The interval lengths should be chosen large enough to assure that there exists enough number of samples at each segment after the length decrement. At least two samples are needed in each segment to perform correct interpolation. For example, if the intervals are segmented to five levels, then the typical threshold length between refined peaks is at least 20 samples. This limits the highest-frequency component that can be used in embedding. For example, if the sampling frequency is $44.1 \mathrm{kHz}$, and the intervals between two successive extrema are at least 20 samples, then the highest-frequency component that is used in embedding is around $1.1 \mathrm{kHz}$. Moreover, if an orthogonal decomposition is applied first, then the subsampling reduces the periods by half at each scale. Hence, 20 samples after two scales of orthogonal decomposition corresponds to a frequency of $1.1 \mathrm{kHz} / 4 \approx$ $275 \mathrm{~Hz}$. For some instruments, these very low frequency components do not exist, and hence the nonorthogonal decomposition should be applied on the original signal directly.

\section{ENCODER/DECODER STRUCTURE}

Due to the complications introduced by the presence of false alarms and missed bits, the encoder/decoder structure of the whole system is more complex than the simple structure described earlier. In this section, we will discuss these structures in detail. In the first subsection, we will discuss the source of false alarms, then we will discuss the encoder/decoder structure to cope with this problem.

\subsection{False alarms}

False alarms pose a serious problem for our algorithm and establish a limitation on the possible embedding rate. These false alarms usually arise after mp3 compression. By false 

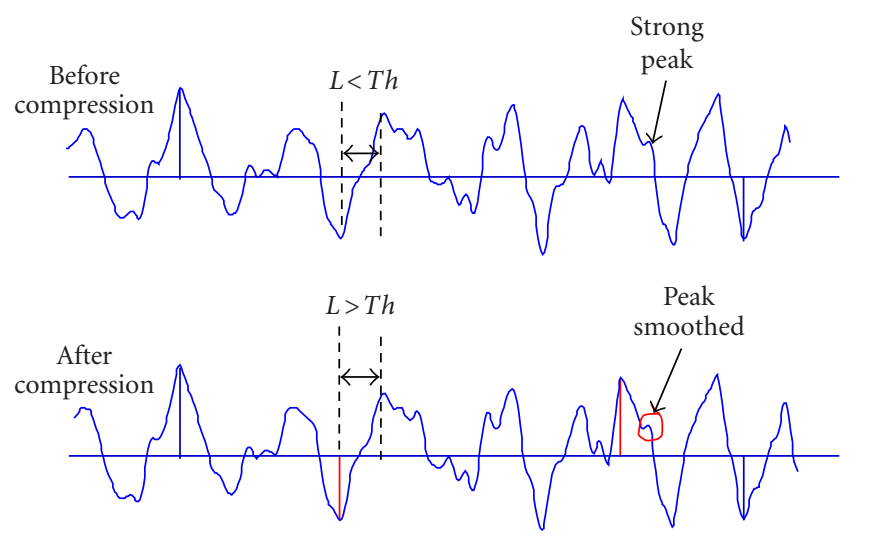

FIgURe 6: False alarms example.

alarms we mean the peaks that are identified by the decoder but not used by the encoder. These false alarms appear because of two main reasons.

(1) The smoothing effect of compression and lowpass filtering. This may remove some weak peaks.

(2) The deviation of some strong peaks at the threshold length after signal processing. For example, assume that refined peaks should be 30 samples apart, then at the encoder peaks that are 29 samples apart are not considered in embedding. However, these periods may increase after compression by one sample (or more). Therefore, the decoder will recognize them as active periods.

These two sources of false alarms are illustrated in Figure 6.

These false alarms lead to a loss in synchronization at the decoder. Remedies for this problem are treated in Sections 4.2 and 4.3. The problem was treated in detail in $[9,10]$.

It should be mentioned that missed peaks might also take place. However, this happens much less frequently than the false alarms. The number of false alarms that arise ranges from $2 \%$ to $15 \%$ of the total number of peaks depending on the nature of the audio signal.

\subsection{Encoding}

To alleviate the problem of false alarms, a self-synchronization mechanism should be contained in the embedded sequence. As mentioned earlier, a repetition code is used at the encoder to improve the convergence and the error performance. If each bit is repeated $r$ times and a single false alarm occurs within a sequence of $r$ similar bits, then it can be easily identified and removed.

The main idea of the encoding algorithm is to isolate the false alarms so as to identify them individually. At each transition from a group of ones to a group of zeros (or the reverse), a marker is put. The sequence of bits between successive markers are decoded separately. In [8], long sequences of zeros or ones are cut by employing high-density bipolar coding (HDBn) scheme in digital communication to add a bit of reverse polarity to a long sequence of similar bits. However, experiments show that this may lead to loss of synchronization in the extracted bits if the extra bit is not identified properly.

\subsection{Decoding}

The decoder performs the following steps.

(1) Extracting the embedded bit as described in Section 2.4. During extraction, each bit is given a score that represents the certainty about the correctness of this bit. The higher the score, the higher the certainty of the corresponding bit. This score is simply the difference between the actual length of the middle segment and the threshold length. These scores are used in further operations.

(2) Applying a median filter (with width $=r$ ) to the extracted bits sequence so as to remove sparse bits that do not agree with their neighbors, and at the same time preserving the correct transition between different bits.

(3) Identifying the markers, which are defined as the points at which a sign change occurs, and the median of its following bits is different from that of the preceding bits.

(4) Identifying the bit sequence between the markers. If the number of bits is divisible by $r$, then the sequence of bits is decoded using the majority rule. The problems arise when the number of bits between two successive markers is not divisible by $r$. For example, assume $r=5$ and the number of bits between two successive markers is 13 , then we have two possibilities. The first possibility is that the correct number of bits is 10 , and we have three false alarms; the other is that the correct number is 15 and we have two missed peaks. The decision between the two possibilities is based on the scores of the residual bits, that is, the three bits with the lowest scores. If the average score of these bits is far smaller than the average of the remaining bits, then they are classified as false alarms, otherwise, they are classified as missed peaks.

(5) Remove the redundant bits that are added at the encoder side if HDBn encoding is employed. This is done by skipping a bit with opposite sign that follows $n$ similar bits in the final output stream.

In what follows, we will discuss the effect of repetition encoding in reducing the probability of false alarms. We will use the following assumptions.

(1) Only false alarms exist (no missed bit).

(2) The probability of false alarms is $P_{f}$.

(3) False alarm events are independent.

(4) Each bit is repeated $r$ times.

(5) All markers are identified correctly.

(6) The number of false alarms between two markers is less than the number of the original bits between them. 
TABLE 1: Probabilities of the number of bits between markers.

\begin{tabular}{ccc}
\hline Bits before encoding & Bits after encoding & Probability \\
\hline 1 & $r$ & $1 / 2$ \\
2 & $2 r$ & $1 / 4$ \\
$\vdots$ & $\vdots$ & $\vdots$ \\
$n$ & $n r$ & $1 / 2^{n}$ \\
$\vdots$ & $\vdots$ & $\vdots$ \\
\hline
\end{tabular}

After repetition, a false alarm exists if there are more than $(r+1) / 2$ false alarms between two successive markers. With repetition, we can have multiple of $r$ bits between two successive markers. If zero and one are equally probable, then Table 1 gives the probabilities for the number of bits between markers.

The number of false alarms within a given number of bits has a binomial distribution because the false-alarm events are independent. The probability of having $k$ false alarms in a sample space of size $N$ bits is

$$
P_{N}(k)=\left(\begin{array}{c}
N \\
k
\end{array}\right) P_{f}^{k}\left(1-P_{f}\right)^{N-k} .
$$

Note that in (1) $N$ takes the discrete values $r, 2 r, 3 r, \ldots$, and so forth. The probability of having a false alarm after encoding is the probability of having $\lceil N / 2\rceil$ false alarms or more between two successive markers, where $\lceil\cdot\rceil$ is the ceiling integer function. Hence the new probability of false alarm is

$$
\begin{aligned}
& P_{F A}=\sum_{m=1}^{\infty} \sum_{k=\lceil m r / 2\rceil}^{m r} P_{m r}(k)\left(\frac{1}{2}\right)^{m}, \\
& P_{F A}=\sum_{m=1}^{\infty} \sum_{k=\lceil m r / 2\rceil}^{m r}\left(\begin{array}{c}
m r \\
k
\end{array}\right) P_{f}^{k}\left(1-P_{f}\right)^{m r-k}\left(\frac{1}{2}\right)^{m}
\end{aligned}
$$

In Figure 7, we show the reduction in the probability of false alarms after using repetition encoding with $n=3,5,7$. Note that, for the typical range of $P_{f}$ (between 0.1 and 0.2 ), the range of $P_{F A}$ is between 0.01 and 0.05 . This range of false alarms is quite adequate for the algorithms described in $[9$, $10]$ to work efficiently with high code rate, for example, $2 / 3$. These algorithms are based on novel decoding techniques for the common convolutional codes.

The overall encoder system consists of a frontend of convolution encoder followed by the repetition encoder which simply repeat each bit for $r$ times. At the decoder side, the repetition decoder (with majority decision rule) is applied on the extracted data, then the convolutional decoder is applied to take care of the residual false alarms. The overall system is shown in Figure 8.

\section{EXPERIMENTAL RESULTS}

The algorithm was applied to a set of 13 audio signals. The lengths of the sequences were around 11 seconds. The test

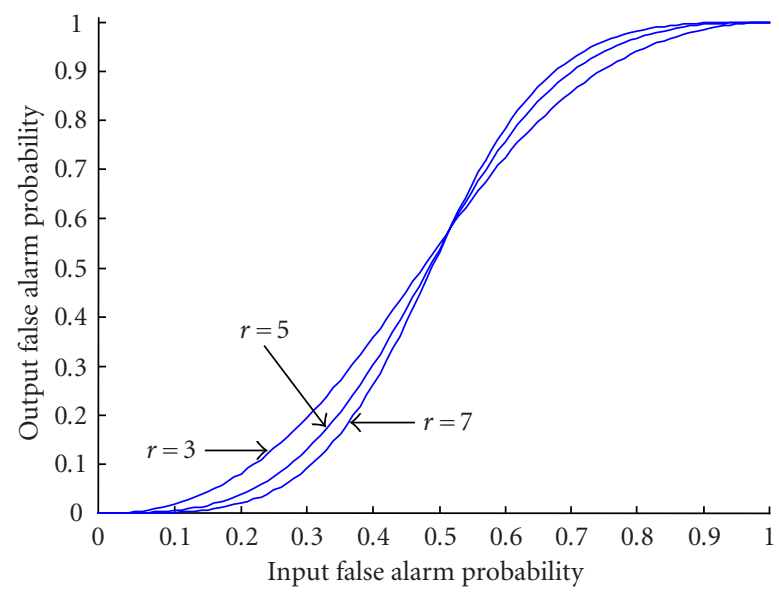

Figure 7: Coding gain after repetition.

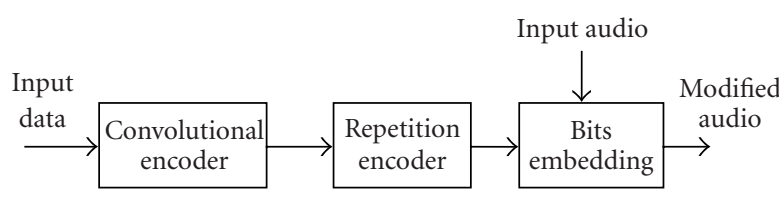

(a) Encoder.

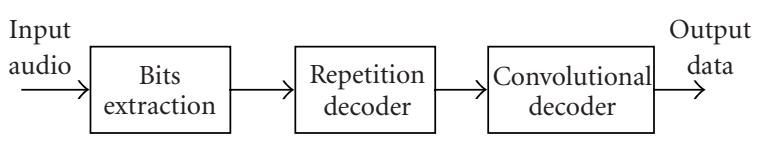

(b) Decoder.

FIGURE 8: Overall encoding/decoding structure.

signals include speech, single instrument music (piano, flute, and violin), and composite music. All test signals are mono with sampling rate $44.1 \mathrm{kHz}$. In all the experiments, we use Daubechies db5 wavelet for orthogonal decomposition, and the derivative of cubic spline wavelet [11] for nonorthogonal decomposition.

The number of levels between two successive extrema is chosen to be an odd number so that the middle segment is usually symmetric around zero. Therefore, the largest modification, which takes place in the middle segment, is in the lowest amplitude region. The typical choice is three or five levels. The larger the number of levels, the better the error performance. However, the output quality (although still high in all cases) is higher with lower number of levels because no large changes occur in this case. It was found that the choice of three levels and interval length of 40 samples gives the best compromise between quality and robustness. This parameter setting is used in all the following tests.

(i) Embedding rate. The median embedding rate of the uncoded data is around 25 bps. However, after coding, the effective embedding rate becomes $5 \mathrm{bps}$. The embedding rate is very large for single instruments, where pure sinusoids with low frequencies are dominant. The 
TABLE 2: Performance versus signal processing operations.

\begin{tabular}{lccc}
\hline Operation & Insertions & Deletions & Errors \\
\hline mp3 compression & 0.039 & 0.0065 & 0.018 \\
LPF $(4 \mathrm{kHz})$ & 0.019 & 0.005 & 0.003 \\
Adding noise $(36 \mathrm{~dB})$ & 0.001 & 0.001 & 0 \\
Resampling to $48 \mathrm{kHz}$ & 0.002 & 0.013 & 0 \\
\hline
\end{tabular}

embedding rates of the algorithm depend heavily on the signal nature. If the signal contains long intervals of low frequencies, then the embedding rate increases significantly. It can be as high as 80 bps for the above parameter setting.

(ii) Noiseless channel. The algorithm described in Sections 2.3 and 2.4 works perfectly with all sequences. However, sometimes, especially with speech signals, it needs an excessive number of iterations at the encoder to converge.

(iii) Quality. The quality of the output signal is very high, and for a nonprofessional listener, it is very hard to distinguish between the original and modified signals. However, when the algorithm was tested with speech signals, the results were not satisfactory.

(iv) Time shift and cropping. The proposed algorithm is automatically robust to time shift and cropping. However, for time cropping, some bits may be missed if a modified interval is cropped. This is unlikely to occur because the intervals used in embedding are usually active audio intervals. If such intervals are cropped, this will affect the audio content. Moreover, with repetition code, deletions can be compensated. However, this can be done only for random deletions. To randomize the effect of time cropping, bit interleaver may be used prior to repetition encoding.

(v) mp3 compression. We tested the performance of the system against $\mathrm{mp} 3$ compression with rates $112 \mathrm{kbps}$ (compression ratio 6.3:1). The average rates are shown in Table 2. These rates are well suited to the algorithm described in [10] to work efficiently. However, at lower compression bit rate, the insertions rate tends to increase significantly.

(vi) Lowpass filtering. Due to the lowpass component of the approximation signal, the algorithm is robust to lowpass filtering. The typical rates are shown in Table 2.

(vii) Time-scale modification. This is the most powerful feature of the proposed algorithm. It is automatically robust to TSM up to a quantization error factor. This means that false alarms (or missed bits) may appear because of the rounding of the thresholds. Consider, for example, if the threshold before TSM is 40 , and the time-scale factor is 0.96 , then the new length becomes 38.4. Then we have two choices for the threshold length (which should be integer), either 38 or 39 . The smaller choice may result in false
TABle 3: Performance versus TSM.

\begin{tabular}{cccc}
\hline Factor & Insertions & Deletions & Errors \\
\hline 0.96 & 0.068 & 0 & 0.018 \\
0.98 & 0.044 & 0 & 0.011 \\
1.02 & 0.023 & 0.006 & 0.006 \\
1.04 & 0.004 & 0.012 & 0 \\
\hline
\end{tabular}

alarms while the larger one may cause missed bits. In Table 3, we show the performance of the algorithm versus different factors of time-scale modifications. In this table, the new threshold length is the round of the old threshold length multiplied by the time-scale factor.

(viii) It should be mentioned that, in the above results, we assumed a fixed time-scale factor. The algorithm can be made robust to time-varying TSM if the threshold of the interval lengths is adaptively updated. From Table 3, it is noticed that either insertions or deletions are dominant at different scale factors. This depends on the rounding. If it is to the smaller integer, then insertions will be more frequent and vice versa. Note that the algorithm is also automatically robust to resampling by any factor. In Table 2 , we show the performance against resampling to $48 \mathrm{kHz}$. It should be mentioned that, for dyadic resampling or upsampling, we may need to reduce the number of decomposition levels at the decoder to match the levels before resampling.

The robustness of the proposed algorithm against mp3 compression and other signal processing operations is comparable to the results reported in recent audio spread-spectrum watermarking works (e.g., $[12,13])$ and projection-based watermarking schemes (e.g., [14]), where the bit error rate is between 0.001 and 0.03 . However, TSM and synchronization attacks have not been studied for most audio watermarking algorithms proposed in the literature because such attacks cannot be compensated within the traditional frameworks. Robustness to these attacks is the main strength of the proposed algorithm.

\section{CONCLUSION}

In this work, we propose a novel algorithm for embedding data in audio by changing the interval length of certain segments of the audio signal. The algorithm is invariant after TSM, time shift, and time cropping. We proposed a set of encoding and decoding techniques to survive the common mp3 compression.

The embedding rate of the algorithm is above 20 bps. However, as discussed for practical reasons, repetition coding is used and the effective embedding rate is $4-8 \mathrm{bps}$. The quality of the output is very high and it is indistinguishable from the original signal. 
The proposed technique is suitable for applications like broadcast monitoring, where the embedded data are information relevant to host signal and used for several purposes, for example, tracking the use of the signal, providing statistical data collection, and analyzing the broadcast content.

\section{REFERENCES}

[1] P. Bassia, I. Pitas, and N. Nikolaidis, "Robust audio watermarking in the time domain,” IEEE Trans. Multimedia, vol. 3, no. 2, pp. 232-241, 2001.

[2] W. Bender, D. Gruhl, N. Morimoto, and A. Lu, "Techniques for data hiding," IBM Systems Journal, vol. 35, no. 3-4, pp. 313-336, 1996.

[3] J. F. Tilki and A. Beex, "Encoding a hidden digital signature onto an audio signal using psychoacoustic masking," in Proc. 7th International Conference on Signal Processing Applications and Technology, pp. 476-480, Boston, Mass, USA, 1996.

[4] S. K. Lee and Y. S. Ho, "Digital audio watermarking in the cepstrum domain," in Proc. IEEE International Conference on Consumer Electronics, pp. 334-335, Los Angeles, Calif, USA, 2000.

[5] D. Kirovski and H. Malvar, "Robust spread-spectrum audio watermarking," in Proc. IEEE Int. Conf. Acoustics, Speech, Signal Processing, vol. 3, pp. 1345-1348, Salt Lake City, Utah, USA, 2001.

[6] M. D. Swanson, B. Zhu, and A. H. Tewfik, "Data hiding for video-in-video," in Proceedings of IEEE International Conference on Image Processing, vol. 2, pp. 676-679, Washington, DC, USA, 1997.

[7] M. F. Mansour and A. H. Tewfik, "Audio watermarking by time-scale modification," in Proc. IEEE Int. Conf. Acoustics, Speech, Signal Processing, vol. 3, pp. 1353-1356, Salt Lake City, Utah, USA, 2001.

[8] M. F. Mansour and A. H. Tewfik, "Time-scale invariant audio data embedding," in Proc. IEEE International Conference on Multimedia and Expo, Tokyo, Japan, 2001.

[9] M. F. Mansour and A. H. Tewfik, "Efficient decoding of watermarking schemes in the presence of false alarms," in Proc. IEEE 4th Workshop on Multimedia Signal Processing, pp. 523528, Cannes, France, 2001.

[10] M. F. Mansour and A. H. Tewfik, "Convolutional decoding for channels with false alarms," in Proc. IEEE Int. Conf. Acoustics, Speech, Signal Processing, vol. 3, pp. 2501-2504, Orlando, Fla, USA, 2002.

[11] S. Mallat, A Wavelet Tour of Signal Processing, Academic Press, Boston, Mass, USA, 2nd edition, 1999.

[12] J. W. Seok and J. W. Hong, "Audio watermarking for copyright protection of digital audio data," Electronics Letters, vol. 37, no. 1, pp. 60-61, 2001.

[13] G. C. M. Silvestre, N. J. Hurley, G. S. Hanau, and W. J. Dowling, "Informed audio watermarking scheme using digital chaotic signals," in Proc. IEEE Int. Conf. Acoustics, Speech, Signal Processing, vol. 3, pp. 1361-1364, Salt Lake City, Utah, USA, 2001.

[14] M. D. Swanson, B. Zhu, and A. H. Tewfik, "Current state of the art, challenges and future directions for audio watermarking," in IEEE International Conference on Multimedia Computing and Systems, vol. 1, pp. 19-24, Florence, Italy, 1999.
Mohamed F. Mansour was born in Cairo, Egypt, in 1973. He received his B.S. and M.S. degrees from Cairo University, Cairo, Egypt, in 1995 and 1998, respectively, and his Ph.D. degree from the University of Minnesota, Minneapolis, Minn, in 2003, all in electrical engineering. During the period 1999-2003, he was with the Department of Electrical and Computer Engineering, University of Minnesota as a Research and Teaching Assistant. In 2003, he joined DSPS R\&D Center at Texas Instruments Inc., Dallas, Tex, as a member of technical staff. His current research interests are in real-time signal processing, adaptive filtering, and optimization.

Ahmed H. Tewfik received his B.S. degree from Cairo University, Cairo, Egypt, in 1982 and his M.S., E.E., and S.D. degrees from the Massachusetts Institute of Technology, Cambridge, MA, in 1984, 1985, and 1987, respectively. Dr. Tewfik has worked at Alphatech, Inc., Burlington, MA, in 1987. He is the E. F. Johnson Professor of Electronic Communications with the Department of Electrical Engineering at the University of Minnesota. He served as a Consultant to MTS Systems, Inc., Eden Prairie, MN and Rosemount, Inc., Eden Prairie, MN and worked with Texas Instruments and Computing Devices International. From August 1997 to August 2001, he was the President and CEO of Cognicity, Inc., an entertainment marketing software tools publisher that he co-founded. Dr. Tewfik is a Fellow of the IEEE. He was a Distinguished Lecturer of the IEEE Signal Processing Society in 1997-1999. He received the IEEE Third Millennium Award in 2000. 